\title{
Design of a Digital Art Teaching Platform Based on Automatic Recording Technology
}

\author{
https://doi.org/10.3991/ijet.v13i08.9050 \\ Wei Liu \\ University of Science and Technology Liaoning, Anshan, China \\ liuwei186cc@126.com
}

\begin{abstract}
Digital media art is a brand-new course combining technology and art under the background of streaming media. Since digital art is based on computer technology, the course is required to keep up with the frontiers of science and technology, and the speed of knowledge update should keep pace with the development of the times. Traditional teaching methods are inflexible and outdated, so that students' knowledge is lagging far behind market demands. In addition, the courseware produced by teachers themselves is not well-targeted and the production cost is high. With this regard, a micro-lesson teaching platform for Digital Art was designed based on the five-star teaching theory and the automatic micro-lesson recording system. The design follows the principles of driving learners' interest with tasks and using learning activities to support services and deepen skills, covering design of micro-lesson learning content, design of learning activities to support services, design of application, and design of evaluation. The results show that the micro-lesson teaching platform can improve learners' interest, achievement and self-confidence in learning; at the same time, it can improve the quality of teachers' micro-lesson resources and reduce the workload of teachers in producing micro-lesson.
\end{abstract}

Keywords-Digital art teaching, Teaching methods, Automatic recording technology

\section{Introduction}

With the rapid development of mobile Internet, people have gradually become accustomed to acquiring information through mobile equipment and learning with fragmented time. Fragmented, mobile, and online learning are changing people's learning modes and lifestyles [1]. In the educational field, micro-lessons as an emerging course form have also experienced rapid development. The teacher's complete explanation of a knowledge point in the teaching process is recorded in a micro-lesson through a video carrier. The duration of a micro-lesson is $5-15 \mathrm{~min}$. The theme of a micro-lesson is usually a special question. Micro-lessons involve an explicit teaching objective, a teaching strategy, and a teaching evaluation system and are characterized by simple examples and concise contents. They can effectively reduce teaching time. Therefore, promoting the classroom teaching level of teachers is essential. 
Only one or two knowledge points are usually explained in a micro-lesson without a complex course system or numerous teaching objectives and teaching objects. A micro-lesson appears to be non-systematic and non-comprehensive. Hence, many call it "fragmentization." However, a micro-lesson targets a specific group and conveys specific knowledge. A micro-lesson should still be systematic, and a group of microlessons still should express all-around knowledge. Through organic integration of micro-lessons, a designer can obtain a micro-lesson teaching platform, which is the foundation of micro-lesson resource construction, sharing, and application. This platform can achieve the integration and screening of micro-lesson resources and offer an application and research environment for users. At present, micro-lesson teaching platforms play an important role in art, history, and other teaching courses [3].

Nevertheless, several problems persist with regard to using micro-lesson teaching platforms. These problems seriously influence the micro-lesson teaching effect. A micro-lesson is not merely a simple video. It should have a clear design sequence and consist of concise PPT, micro-lesson subject with an explicit theme, title with certain information, main content with logic, and tail leader with a guidance function. Many difficulties exist in micro-lesson video recording, and these also influence the microlesson teaching effect. Given such a background, automatic recoding theology was applied in this study to design a digital art micro-lesson teaching platform for the provision of new insights into micro-lesson production.

\section{State of the art}

Micro-lessons emerged and developed rapidly around 2010. Foreign theoretical studies on micro-lessons began early, but these studies are few in number, and a complete research system has not been formed. Sánchez et al. [4] compared the perceptions of students who provide instructions for tissue engineering by microteaching with the perceptions of the same students receiving such instructions. The results indicated that microteaching-based self-assessment related to feedback on selflearning renders microteaching a useful technique for self-learning and a crucial tool for promoting self-regulation in the course. At the National Institute of Education in Singapore [5], a micro-lesson was introduced into the instructional technology course of the teacher education program to provide opportunities for pre-service teachers to apply constructivist instructional approaches in the design of multimedia learning packages and discuss how to incorporate micro-lesson teaching into the pre-service teacher training program. Domestic studies on micro-lesson design and application have focused on courses in middle and primary schools, but extension to higher education is imminent. Wei [6] selected vocational college students as the object of study and designed and completed an art course learning platform based on micro-lessons by combining the psychological features and learning needs of this group. The platform can operate in mainstream mobile terminals, is in accordance with microlearning needs, and provides necessary support for art teaching reform. Zhang [7] comprehensively analyzed the features of web system development technology and proposed a new teaching mode that combines traditional and micro-lesson learning. 
On this basis, a corresponding micro-lesson teaching platform was developed, and empirical research was performed on the platform.

Micro-lesson recording has been a focus of domestic and overseas scholars. These scholars have exerted extensive efforts to produce and develop improved microlessons. Horiuchi et al. [8] compared and analyzed the advantages and disadvantages of micro-lesson reduction methods, such as external video shooting, computer screen recording, and panel recording. They adopted the Khan Academy model and used mathematics micro-lessons for primary schools as an example. According to them, topic features, hardware conditions, and software operation level should be considered when selecting a micro-lesson recording method. The authors in [9] indicated that although many micro-lesson teaching cases and videos have emerged recently, most of them do not conform to quality standards. The key to micro-lesson production with the main manifestation pattern of micro-video is video recording. They proposed the idea of producing micro-lesson videos with automatic recording and playing and optimized micro-lesson production from preparation before recording to the recording process and editing in the late stage.

This new teaching form (i.e., micro-lessons) is generally believed to exert a positive effect on traditional teaching. New techniques are continuously introduced to optimize the production micro-lessons. The practice of micro-lesson teaching has also intensified. High-level, high-quality micro-lesson teaching resources emerge continuously. However, these numerous micro-lesson teaching resources have two primary deficiencies. First, only the content presentation form is valued in micro-lesson production, and the teaching design guided by teaching principles is disregarded. This disregard severely affects the teaching efficiency of micro-lesson resources [10]. Second, the teaching design ideas of diverse micro-lessons differ considerably. Micro-lesson constructors cannot easily refer to previous materials. Moreover, continuous improvement is required in terms of standardizing the production flow and simplifying the recording technology. From the technological aspect, the basic microlesson recording method is the camera and recording system. Editing is then conducted with a nonlinear editing software to form a micro-lesson [11]. This method is complex and consumes much time. The required operations are difficult to control, and the production cost is high. The present study used five-star teaching theory and automatic micro-lesson recording technology to solve these problems and improve teaching efficiency by utilizing the advantages of mobile Internet and mobile equipment.

This research aims to solve the theoretical inadequacy and technological immaturity of the micro-lesson teaching platform. Five-star teaching theory was introduced to offer teaching method guidance for micro-lesson teaching design. The micro-lesson design process was standardized, and the teaching efficiency of micro-lesson resources was improved by activating learned knowledge, demonstrating new knowledge, trying applications, and digesting. Furthermore, automatic recording technology was utilized to replace the camera and teaching recording system and thus resolve the issues of long production period and complex process. 


\section{Digital art micro-lesson teaching platform based on automatic recording technology}

The deficiency of instructional theory results in disordered micro-lesson teaching platform content, low pertinence, and non-ideal teaching effect. The immaturity of recording technology also makes the quality of micro-lessons uneven. To solve these problems, theory construction was conducted to create a micro-lesson teaching platform based on automatic recording technology.

\subsection{Micro-lesson teaching design based on five-star teaching theory}

Information presentation is valued in online and multimedia teaching, and effective teaching is ignored. Five-star teaching theory [12] was proposed for this problem. The core of this theory includes the following: involve learners in practical problems, activate learned knowledge and regard it as the foundation of new knowledge, demonstrate new knowledge to learners, let learners apply new knowledge, and link new knowledge with learners' life. These tasks can promote learning. The pattern of five-star teaching theory is shown in Fig. 1.

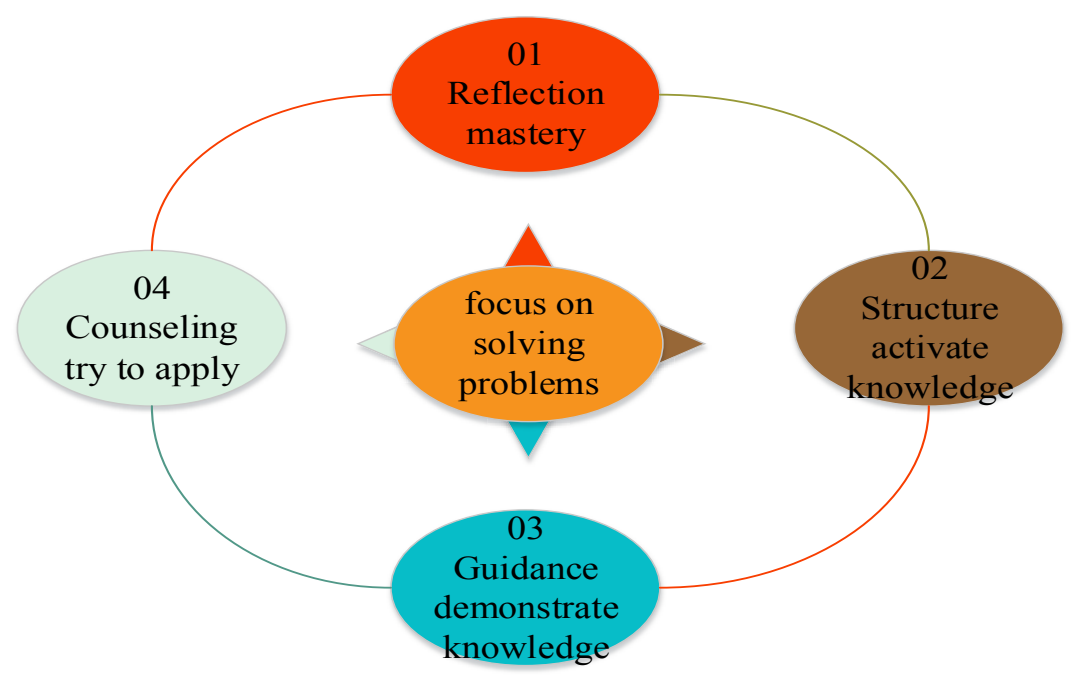

Fig. 1. Mode pattern of five-star teaching theory

As indicated in the figure, the core of five-star teaching theory is "focus on problem solving." Its inner layer is a teaching process involving the following stages: "activate learned knowledge $\rightarrow$ demonstrate new knowledge $\rightarrow$ try application $\rightarrow$ digest." The outer layer is a learning process that consists of "structure $\rightarrow$ guidance $\rightarrow$ instruction $\rightarrow$ reflection." In five-star teaching theory, a specific teaching task is completed by gradual problem solving, that is, present the question to learners, carry out teaching according to the teaching tasks, and show how to apply the knowledge to 
solve problems or complete tasks. Micro-lesson design should be in accordance with five-star teaching theory to conform to the learning process and psychological development of learners. A micro-lesson teaching design mode based on five-star teaching theory is shown in Fig. 2.

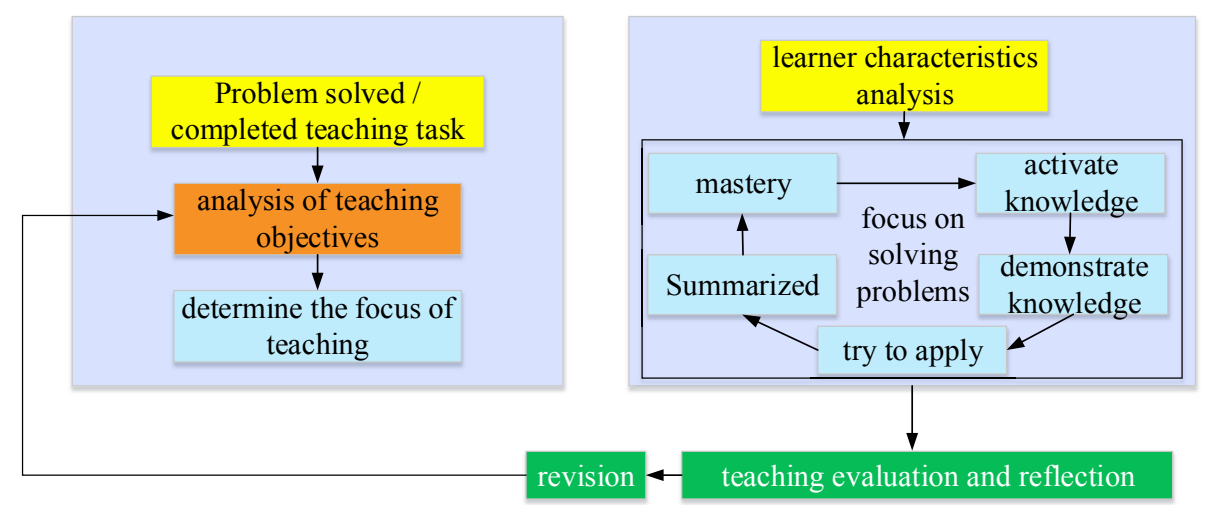

Fig. 2. Micro-lesson teaching design framework based on five-star teaching theory

The key design points of each link in the teaching circle are as follows:

(1) Active learned knowledge: At least two aspects of teaching activities should be designed in a micro-lesson. First, the learned knowledge diagram can stimulate and guide learners to recall. Second, rich teaching experience is used to help learners transition to the current learning content.

(2) Demonstrate new knowledge: This part is the main body of micro-lesson content, and it consists of two sub-parts. The first one is new knowledge organization and exhibition method design. Different teaching contents can be matched with corresponding presentation methods according to knowledge type, including positive and negative cases, logic progression, and examples. The second one is media selection and design. The teacher should choose suitable media to promote learning according to the content type. Media matching should not be based on diversity but should focus on applicability. The last sub-part is learning guidance design. Learning guidance should be integrated into the micro-lesson content. Close-up shots and media materials should be adopted to enhance the learning of key and difficult knowledge.

(3) Try application: The teacher should apply guiding practice and tests to provide opportunities for students to try the application of new knowledge and new skills. The design applied should guarantee content relevance, and the content should be set according to the difficulty.

(4) Summarize: Summarization of different knowledge modules is an effective means to integrate new knowledge into the existing knowledge structure of learners. The teacher should accurately generalize knowledge instead of repetition.

(5) Digest: When the micro-lesson ends, the content should include assignment arrangement and after-class thinking. The topic should be the original situation with proper changes to test the ability of learners to solve problems. 
In traditional micro-lesson recording processes, a camera and a teaching recording system are used to record the course content. Then, a nonlinear editing software is applied to edit the video and complete the micro-lesson. This method is time consuming and labor intensive. Adapting to micro-lesson production requirements in the Internet era is also difficult. A fully automatic micro-lesson recording system based on automatic recording technology was developed in this work to simplify the microlesson recording process. The system diagram is shown in Fig. 3.

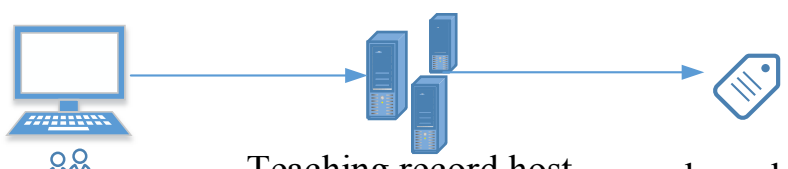

micro-class controller

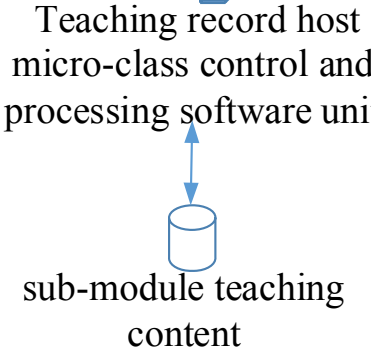

$$
\begin{aligned}
& \text { knowledge } \\
& \text { matching module }
\end{aligned}
$$

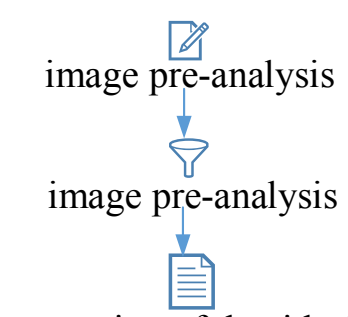

extraction of the title from the file

Fig. 3. Automatic recording system based on automatic recording technology broadcast system block diagram

The fully automatic micro-lesson recording system is composed of a micro-lesson controller, a teaching recording machine, a knowledge point matching module, and a teaching content sub-module. The micro-lesson control treatment software unit is set in the teaching recording machine. The system operates as follows:

First, the micro-lesson controller triggers micro-lesson recording and sends the instruction to the micro-lesson processing unit of the teaching recording machine through WiFi or RS232. The instruction includes the course number.

Second, the micro-lesson mode recording begins after the micro-lesson control processing software unit receives the signal. Computer and teacher camera pictures are obtained, and the pictures are composited by applying the picture-in-picture mode. In the meantime, the signal is sent to the teaching content submodule.

Third, the teaching content submodule starts to analyze the content after it receives the signal. The module decides whether to start pre-selected knowledge point extraction according to the foundation of image pre-analysis. The title is extracted from PPT files or video frames. The content with a type size greater than the average type size and $1 / 3$ of the image is extracted from the image according to the type size and location features. 
Fourth, the knowledge point matching module executes knowledge point matching. The course knowledge point set is searched in the knowledge point database according to pre-selected knowledge point information. Then, a matching operation is conducted. When the knowledge points are updated and the last knowledge point continues to be recorded for over $5 \mathrm{~min}$, the knowledge point matching module sends the signal of competing current recording to the micro-lesson control processing software unit.

Finally, the micro-lesson control processing software unit receives the signal of competing current recording, begins to analyze the information of the next knowledge point, and saves the current micro-lesson. Meanwhile, a new knowledge point recording process starts, and the entire process of fully automatic micro-lesson recording is completed. The interface of the fully automatic micro-lesson recording system is shown in Figs. 4 and 5.

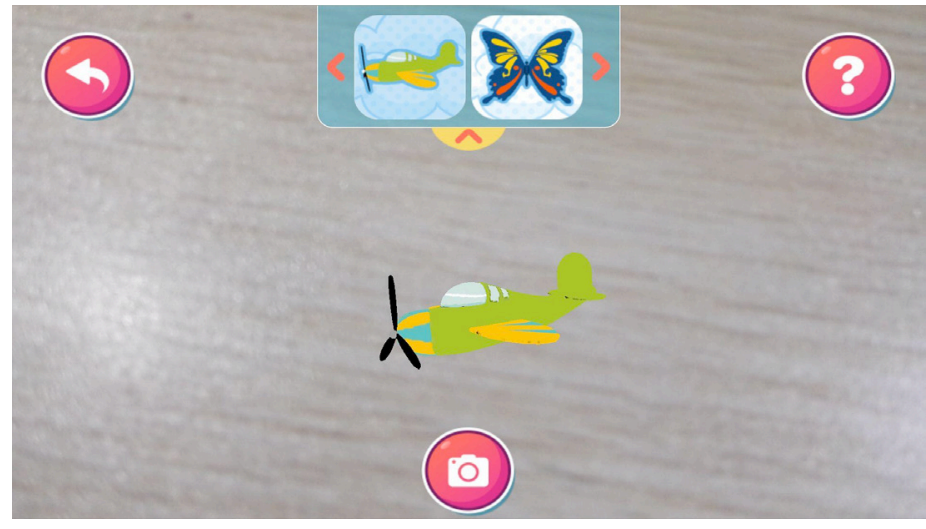

Fig. 4. Interface 1 of full-automatic micro-lesson recording system based on automatic recording technology

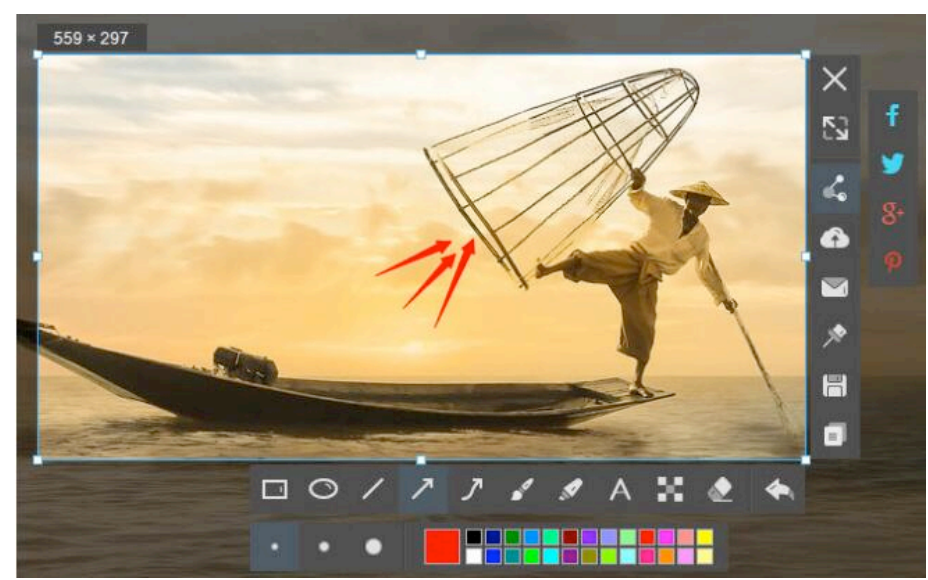

Fig. 5. Interface 2 of full-automatic micro-lesson recording system based on automatic recording technology 


\section{$4 \quad$ Teaching example and teaching effect}

\subsection{Teaching example}

On the basis of five-star teaching theory and the fully automatic micro-lesson recording system, a digital art micro-lesson teaching platform was designed by following the principles of task-driven learners' interest and service deepening support through learning activities. The design included four aspects: micro-lesson learning content design, learning activity support service design, application method design, and evaluation design. The entire framework of the digital art micro-lesson teaching platform is shown in Fig. 6.

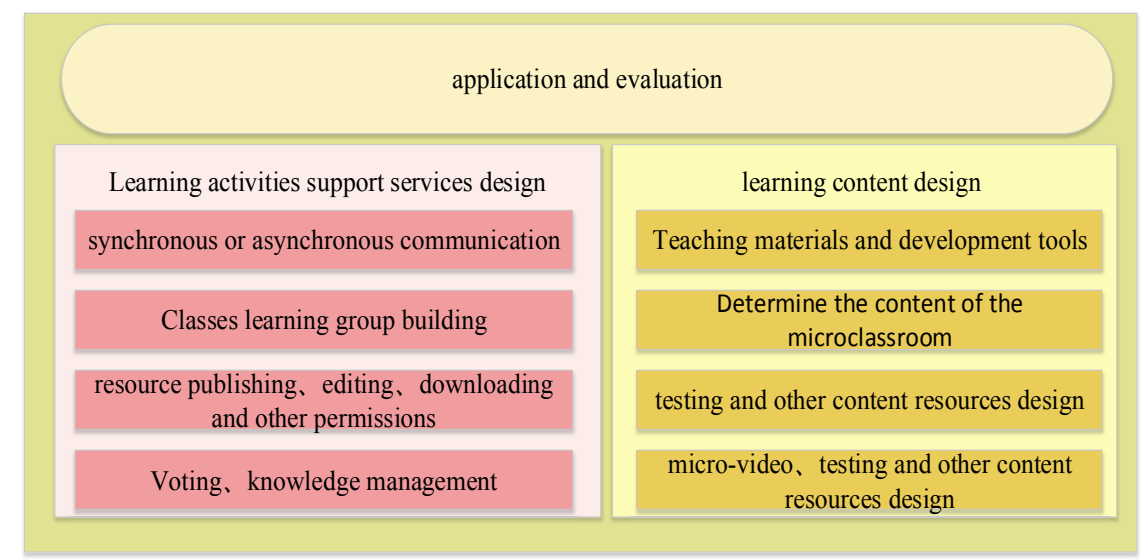

Fig. 6. Entire framework of Digital Art micro-less teaching platform

1. Learning content design: The micro-lesson objective was confirmed via teaching analysis. Learners were required to know the skills they should have after learning. Next, the micro-lesson was confirmed, and the knowledge granularity of the teaching content was classified. The content of a single micro-lesson should be targeted and focus on a prominent knowledge point. Knowledge integrity must also be ensured. Then, available high-quality materials and learning resources were selected for resource integration and recreation. The micro-lesson content was organized and arranged in accordance with certain technical standards.

2. Learning activity support service design: Suitable learning auxiliary tools were matched according to different course features, including voting, knowledge management, resource release, modification, and downloading rights.

3. Application method design: After the micro-lesson resources were published on the micro-lesson teaching platform, they were applied through in-class hierarchical teaching and after-class instruction. The in-class hierarchical teaching link design is shown in Fig. 7. 

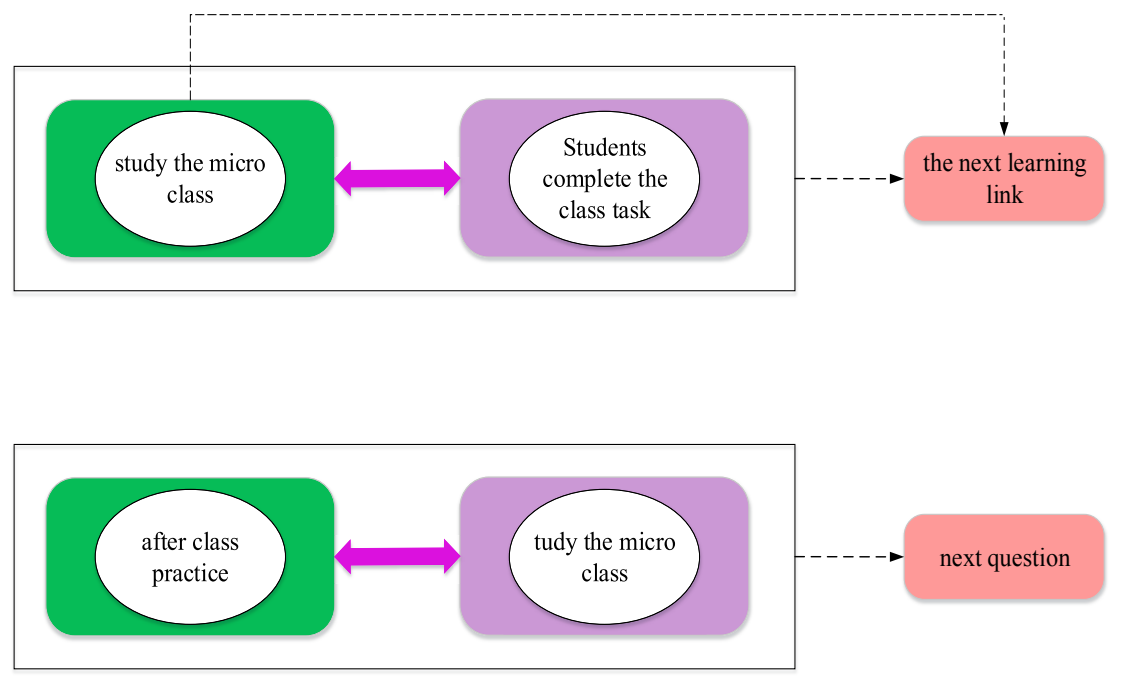

Fig. 7. In-class hierarchical teaching link design

In the teaching process, after explaining a knowledge point, the teacher would assign a practical training exercise to test the learning effect. For students with poor test results, the teacher could arrange micro-lesson learning again to review relevant knowledge points. Students with outstanding abilities could improve their knowledge level by expanding learning resources or entering the next link in advance.

According to the problems proposed by students in class, the teacher could analyze the concepts and principles involving the problems for micro-lesson design to meet students' autonomous learning needs.

(4) Evaluation design: The micro-lesson evaluation system included effect evaluation and micro-lesson design effect evaluation [7]. Learning effect evaluation is the most important evaluation criterion for micro-lessons. The teacher can combine knowledge tests, skill operations, and learning experiences to evaluate the learning effect, accurately understand a learner's performance and output in micro-lesson learning, and verify the effectiveness and practicability of the micro-lesson. Evaluation of the micro-lesson design effect was conducted with an evaluation scale. The dimensionality setting is shown in Table 1.

Table 1. Evaluation of micro-lesson design effect

\begin{tabular}{|c|l|}
\hline Dimensionality & \multicolumn{1}{c|}{ Variable } \\
\hline Teaching & $\begin{array}{l}\text { Teaching objective, teaching strategy, teaching content, teaching arrangement, teach- } \\
\text { ing method, learning evaluation, micro-video production }\end{array}$ \\
\hline Interaction & Student-teacher interaction, learner-media interaction \\
\hline
\end{tabular}

The information about micro-lesson design effect evaluation could be fed back to micro-lesson design link timely to make it perfect continuously. Teaching link situation of micro-lesson teaching platform is shown in Fig.8. 


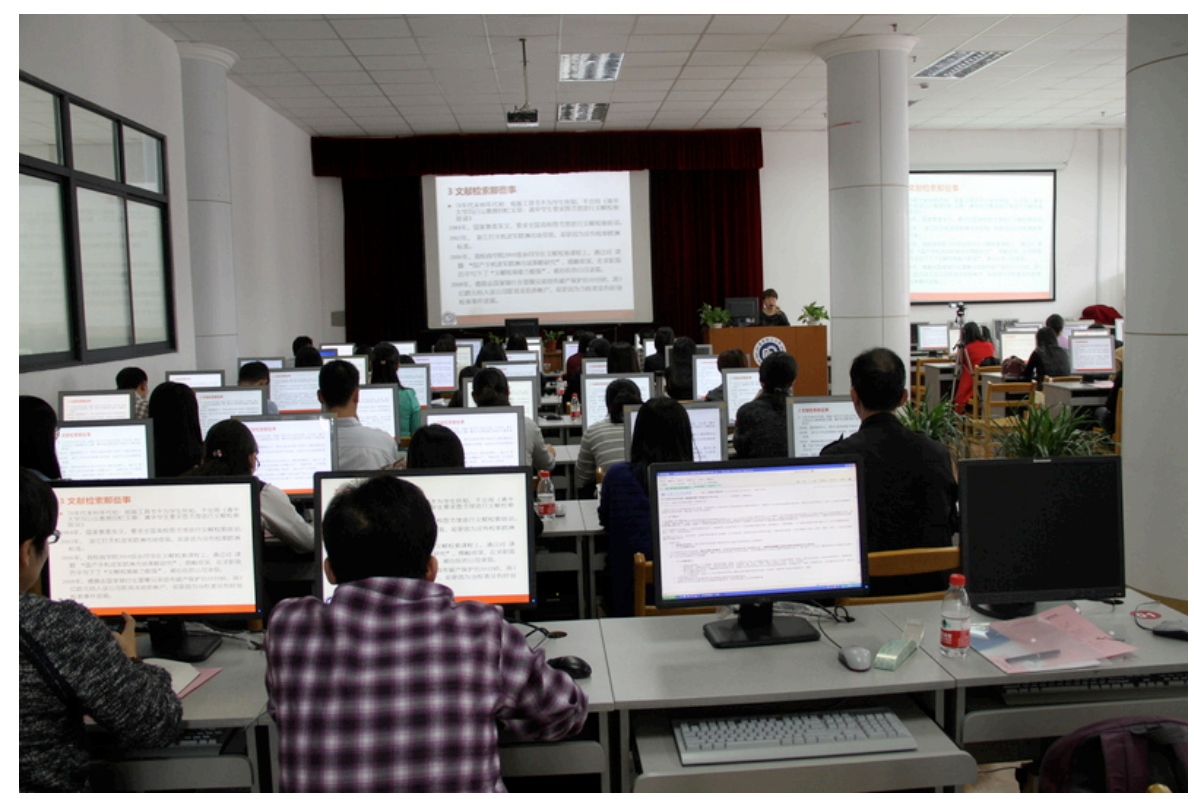

Fig. 8. Student-teacher interaction process diagram of full-automatic micro-lesson teaching mode

\subsection{Teaching effect}

Digital art applies digital technology and computer programs to analyze and edit pictures and videos for further upgrading. Digital art exhibits irreplaceable advantages in terms of communication, storage, and replication. Digital art has penetrated all walks of life due to the rapid development of computer technology. Given that a digital art course integrates interactive media design, virtual reality design, and new media art, high requirements are set for learners' learning and operational abilities. Low teaching efficiency is commonly observed in digital art course teaching. The introduction of the micro-lesson teaching platform is crucial in improving the teaching efficiency of digital art.

Digital art was selected as the experimental course in this work. The total period was $32 \mathrm{~h}$. One hundred sophomores of the digital media art major in a college were chosen as the experiment objects. The learning content included theory and practice. The cooperation between researchers and practitioners was the foundation for implementing the experiment in an orderly manner. In the experiment process, the experiment object adopted the micro-lesson teaching platform to supplement classroom study and carry out after-class instructions. Students could browse the automatically recorded teaching videos and fed back the problems to the teacher through the platform. After the course ended, teaching effect evaluation was conducted through the micro-lesson teaching platform, and the questions put forward by the platform were answered. The micro-lesson teaching platform integrated the learners' evaluation and analyzed the application effect according to statistical data. 
Two problems were investigated, and the results for both are shown in Table 2. The two problems were as follows: whether the micro-lesson teaching platform can promote learners' learning interest and whether micro-lesson learning can enhance learners' learning achievement and self-confidence.

Table 2. Influence of Micro-learning on Learners

\begin{tabular}{|l|c|c|c|c|}
\hline & $\begin{array}{c}\text { Greatly } \\
\text { improved }\end{array}$ & Improved & $\begin{array}{c}\text { Little im- } \\
\text { proved }\end{array}$ & No improved \\
\hline Learning interest & $20(20 \%)$ & $40(40 \%)$ & $35(35 \%)$ & $5(5 \%)$ \\
\hline Achievement and confidence & $35(35 \%)$ & $50(50 \%)$ & $5(5 \%)$ & $10(10 \%)$ \\
\hline
\end{tabular}

Table 2 shows that among the students participating in the experiment, $20 \%$ believed that the micro-lesson teaching platform considerably promoted their learning interest, $40 \%$ chose "improvement," and 35\% considered the learning general. Overall, the micro-lesson teaching platform can promote the learning interest of most learners. Table 2 also shows that over $80 \%$ of the learners believed that micro-lesson learning improved their learning achievement and self-confidence, and only $10 \%$ chose "no improvement."

The results indicated that the micro-lesson teaching platform, which can improve learners' learning interest, learning achievement, and self-confidence, was welcomed by the students. Hence, it can supplement classroom teaching. The reasons are as follows. First, this research based on the core concept of five-star teaching principle guides micro-lesson teaching design practice. The practice focuses on the central problem with a task feature. Micro-lesson teaching was organized in five stages: activate learned knowledge $\rightarrow$ demonstrate new knowledge $\rightarrow$ try application $\rightarrow$ summarize $\rightarrow$ digest. For example, for "try application," the teacher adopted an incentive mechanism in the scoring process aside from taking care of the students' practical ability. For original and excellent works, the score is high. For works that quote materials, the score is relatively low. This scheme aims to encourage students to think, practice, and better complete the works. For "summarize," the teacher and students appreciated the works together, summed up the experiment process, analyzed the advantages and disadvantages of the works by adopting theoretical knowledge on digital media, and determined the gap with excellent digital media works. In this manner, the cognition structure of students can be constructed, enriched, and promoted gradually. This teaching method can be used for instruction-type micro-lessons or other types of micro-lessons. With its good teaching property, effectiveness, and universality, this teaching method can fully promote the learning participation of learners, guide learners as they conduct positive meaning construction for knowledge, and facilitate independent study and innovation ability improvement among learners. Furthermore, automatic recording technology can ensure the quality of micro-lesson resources. For example, it guarantees picture smoothness of live micro-lessons, avoids wasting learners' time, reduces the workload for teachers in micro-lesson production, and further ensures teaching quality and effect. It also provides necessary technical support for further popularizing micro-lesson teaching platforms. 


\section{Conclusions}

A micro-lesson is a mini-course in which learning activity support service is added on the basis of micro resources. It is a carrier of content, service, and interaction. Practice has proven that a micro-lesson teaching platform based on automatic recording technology exerts a remarkable effect on digital art teaching. The simplicity and high efficiency of micro-lessons greatly promote the development of multimedia teaching. From the technical aspect, a micro-lesson teaching platform can achieve fully automatic recording of micro-lesson videos, thus ensuring the quality of microlesson resources. From the theoretical aspect, a micro-lesson teaching platform achieves normative design of micro-lesson teaching. The application of micro-lesson teaching platforms in digital art teaching can facilitate the popularization of the micro-lesson teaching mode, and it plays a positive role in college teaching.

\section{Acknowledgment}

This work was supported by 2013 General Project for the 12th Five-Year Educational Science Plan of Liaoning Province (JG13DB085) and 2016 General Project for Higher Education's Association "13th Five-Year Plan" of Liaoning Provincial (GHYB160154).

\section{$7 \quad$ References}

[1] Brown, A., Kappes, J., Marks, J. Mitigating theme park crowding with incentives and information on mobile devices. Journal of Travel Research, 2013, vol. 52(4), pp. 426-436. https://doi.org/10.1177/0047287512475216

[2] Lim, C.P., Chan, B.C. microLESSONS in teacher education: Examining pre-service teachers' pedagogical beliefs. Computers \& Education, 2007, vol. 48(3), pp. 474-494. https://doi.org/10.1016/j.compedu.2005.03.005

[3] Zhang, F. Quality-Improving Strategies of College English Teaching Based on Microlesson and Flipped Classroom. English Language Teaching, 2017, vol. 10(5), pp. 243-245. https://doi.org/10.5539/elt.v10n5p243

[4] Sánchez, A.C., Ferrer, P.V.C., Alaminos, M. Microteaching as a self-learning tool. Students' perceptions in the preparation and exposition of a microlesson in a tissue engineering course. Journal of Technology \& Science Education, 2013, vol. 3(2), pp. 66-72.

[5] Alerić, M., \& Prskalo, R. Attitudes of students at teacher education institutions towards the standard language and deviations from the morphological norm. Odgojne Znanosti, 2012, vol. 14(1), pp. 460-462.

[6] Wei, N.B. Research on the Application of Micro-lesson in the Course of Database Technology. Education Teaching Forum, 2017, vol. 13, pp. 266-267

[7] Zhang, E., Zhang, W. Construction and Application of MOOC-based College English Micro Lesson System. International Journal of Emerging Technologies in Learning, 2017, vol. 12(2), pp. 155-157.

[8] Horiuchi, K. Effects of Micro-teaching in Teacher Training Class of Junior High School Home Economics Education: focusing on the interactive evaluation by"comment reports". 
Journal of the Japan Association of Home Economics Education, 2008, vol. 51, pp. 169179.

[9] Yuan, X.H. The Application of Microteaching Mode in Primary School Chinese. Education Teaching Forum, 2014, vol. 12, pp. 81-82.

[10] Compton, L., \& Davis, N. The impact of and key elements for a successful virtual early field experience: lessons learned from a case study. Contemporary Issues in Technology \& Teacher Education, 2010, vol. 10(3), pp. 309-337.

[11] Souers, C.V., Winslow, D.R. Ideas to Stimulate Lessons in Measurement for Upper Elementary and Junior High School Students. School Science \& Mathematics, 2015, vol. 66(6), pp. 532-534. https://doi.org/10.1111/j.1949-8594.1966.tb13582.x

[12] Jiang, Y.B., He, Y.H., Yang, C.X. Teaching Exploration of Structural Stability Curriculum with Five Star Principles of Instruction. Journal of Architectural Education in Institutions of Higher Learning, 2008, vol. 17(1), pp. 51-54.

\section{Author}

Wei LIU is a lecturer in the School of Architecture and Art Design, University of Science and Technology Liaoning, Anshan, China (liuwei186cc@126.com).

Article submitted 26 February 2017. Resubmitted 28 May 2018. Final acceptance 03 July 2018. Final version published as submitted by the author. 